\title{
SYNDROME OF INCOMPLETE REGIONAL ACHONDROPLASIA (ILIUM AND RIBS) WITH ABDOMINAL MUSCLE DYSPLASIA
}

BY

\author{
EMMANUEL SHAPIRA, EFRAIM FISCHEL, SHIMON MOSES, and STANLEY LEVIN \\ From the Department of Pediatrics B and Radiological Department, Kaplan Hospital, Rehovoth, Israel
}

(RECEIVED FOR PUBLICATION APRIL 6, 1965)

The classical picture of achondroplasia with abnormal formation of bone from cartilage involves mainly the long bones, and is well described in medical literature. In its incomplete form, the disease has been reported as affecting one side of the body, one extremity, or even a single bone (Caffey, 1948). We have recently come across a case of an infant with regional achondroplasia of only the pelvis and ribs, together with pronounced weakness of the abdominal muscles. In this child's family the syndrome is fully encountered in the proband, and appears partially in several others.

\section{Case Report}

K.M. was born following an uncomplicated pregnancy and delivery, weighing $3 \cdot 2 \mathrm{~kg}$. She was first admitted to our department on the day after birth because of marked deformity of the chest and suspected congenital lack of abdominal muscles. The outline of the stomach and intestines were very apparent, and peristalsis could easily be seen through the skin of the abdomen. On subsequent admissions, usually due to recurrent respiratory tract infections, gradual development of the abdominal muscles was observed, though, when last examined at the age of 4 years, a considerable degree of hypoplasia was still evident together with a muscle defect in the area between the rectus abdominis and obliquus externus muscles on the left side. There were umbilical and bilateral inguinal herniae. The child had always been small for her age, of asthenic build with narrow chest, wide at the base and tapering towards the apex. The abdomen was protuberant (Fig. 1a and b). Mental development appeared to be entirely normal.

Laboratory Findings. Haemoglobin and differential blood counts were found to be normal on successive examinations. Blood proteins, glucose, and urea were within normal limits as were calcium, phosphorus, and phosphatase.

X-ray Examinations. Repeated radiological examinations of the skull, limbs, and vertebrae showed no abnormalities. The pelvis and ribs, however, showed the typical deformities of achondroplasia (Caffey, 1958). In the radiological examination carried out at the age of 3 days, the lower part of the ilium was missing. The acetabular angle of inclination was found to be zero (normal $28^{\circ}$ ), and the greater sciatic notch was deep and narrow. At the ages of $2 \frac{1}{2}$ and 4 years, progressive development of the pelvic bones was plainly discernible (Fig. 2a, b), though the above-mentioned deformity was still present. In addition, an almost complete closure of

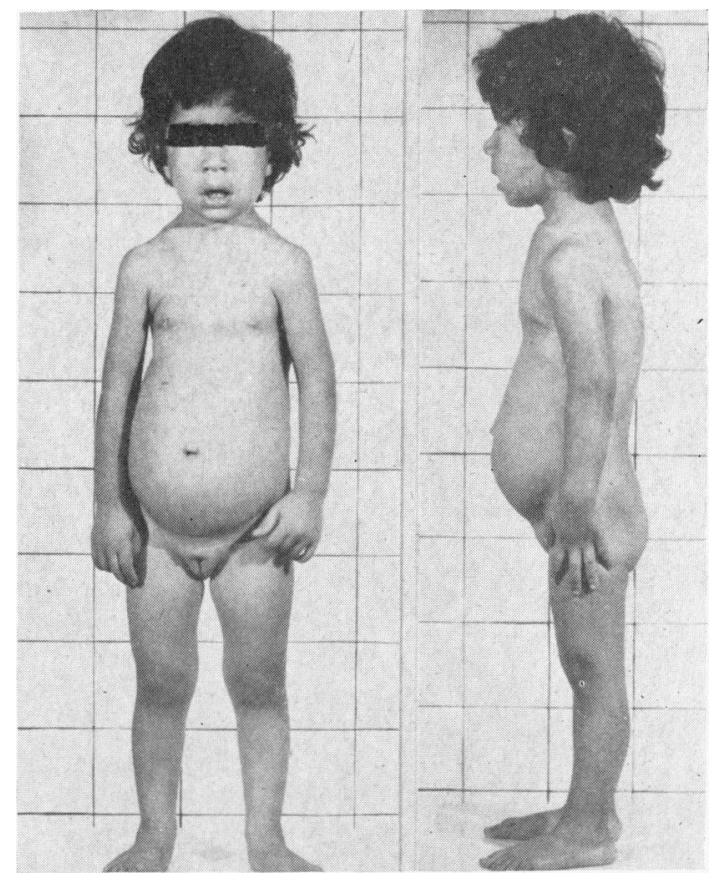

FIG. 1a, b.-A child with achondroplasia of pelvis and ribs and hypoplastic abdominal musculature at the age of $2 \frac{1}{2}$ years. Note protuberant abdomen, short pelvis, deformed chest, and normal body limb proportions. 


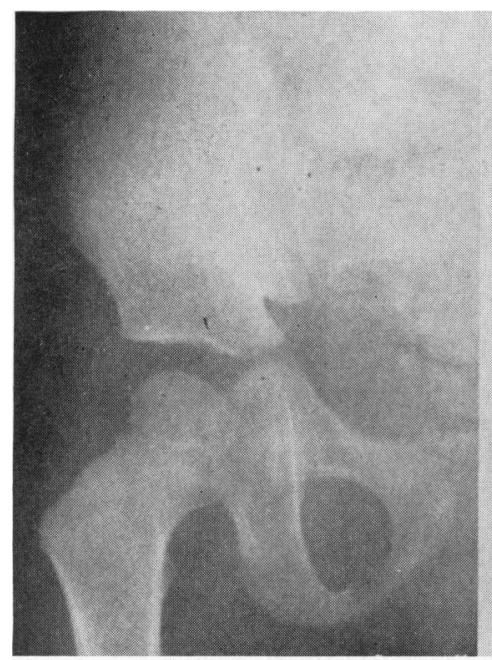

(a)

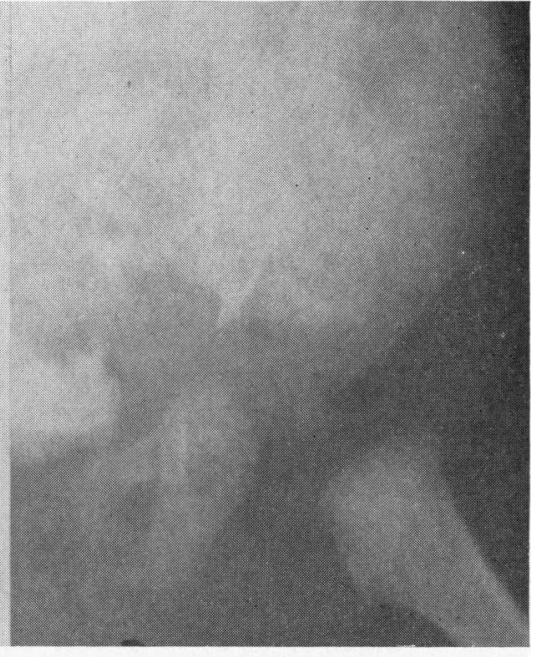

(b)

FIG. 2.-Radiographs showing typical achondroplastic changes in the pelvis: (a) at birth; (b) at $2 \frac{1}{2}$ years.

the ischio-pubic synchondrosis was present at the age of $2 \frac{1}{2}$ years (normal at about 6 years). The chest radiographs showed deformity of all ribs with flattening of the tips, which accounted for the chest deformation as a whole (Fig. 3a, b). Intravenous pyelography on two occasions was normal.

Family History. The parents were first cousins (Fig. 4). On the father's side (III. 4-16), 3 brothers and 2 sisters died in childhood of unknown causes. Another brother (III. 6) died of congenital heart disease at the age of 22 years. There were also two spontaneous miscarriages. In the patient's generation (IV. 1-8), one sister and one brother (IV. 4,6$)$ died on delivery and another child died on the 6th day of life. In these last three cases a good many skeletal deformities were noticed, one infant having 'hydrocephalus'. Two pregnancies of the patient's mother ended in spontaneous abortions.

Of 16 members of this family (II, III + IV) surviving infancy, 8 had either umbilical, femoral, or inguinal herniae. 3 had chest deformities similar to those of the patient (IV. 3 and III. 6, 12), though in 2 of them, an aunt and an elder sister of the patient, chest and pelvic radiographs showed no abnormalities.

Details of generation I and the offspring of II.1 are unknown.

\section{Discussion}

Whereas achondroplasia in its classical form has been found in a skeleton of the Neolithic period some 7,000 years old, as well as in pictures of ancient Egyptian gods, it was Parrot in 1878 who described it clearly as an entity for the first time.

Among the known cartilaginous dysplasias, achondroplasia is the most frequent, being in fact a hypoplasia and not an aplasia. The pathological

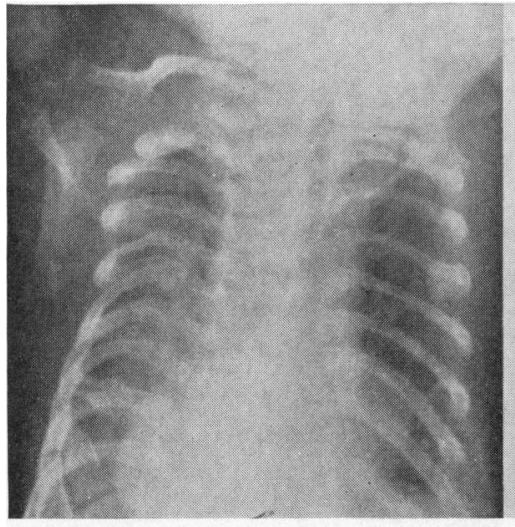

(a)

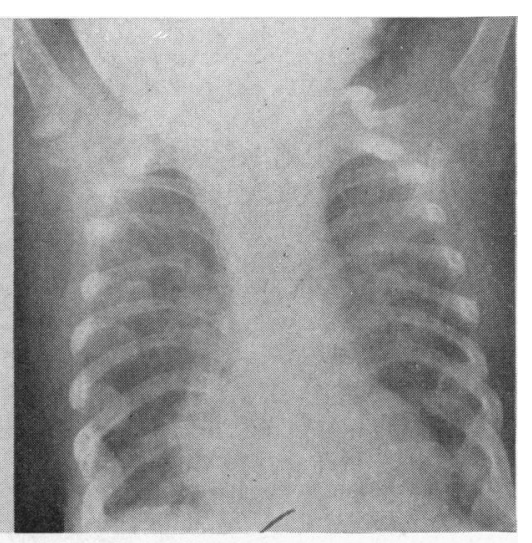

(b)

FIG. 3.-Radiographs showing achondroplastic changes in the ribs: (a) at birth; (b) at $2 \frac{1}{2}$ years. 


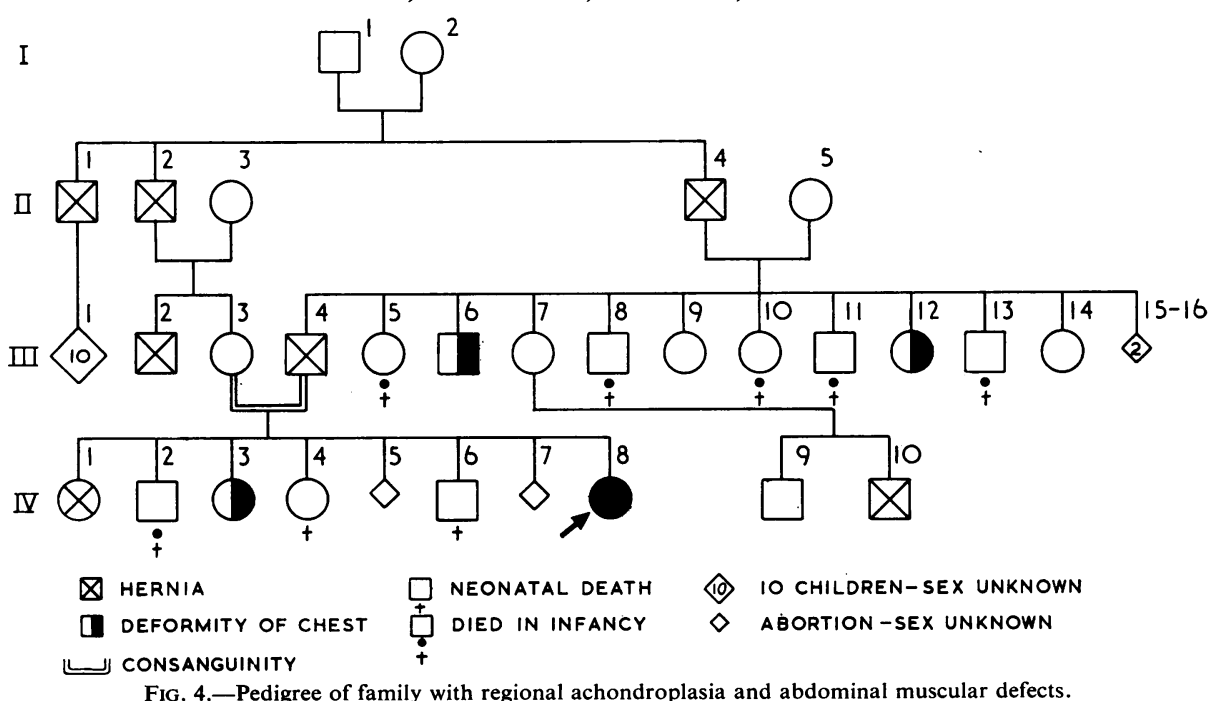

picture indicates a defect in cartilaginous growth and in bone formation from cartilage (Lane, 1960).

In its complete form the disease affects mainly the long bones. Other bones involved are the ribs, vertebrae, scapulae, skull-particularly at its base, and pelvis-particularly the ilium. For patients surviving the first year of life, prognosis is favourable.

In our case, the disease affected only the pelvis and ribs and was accompanied by congenital hypoplasia of the abdominal muscles. The radiological picture of the pelvis was identical to that described by Caffey in 1958 (Fig. 5): on studying the radiographs, he expressed the opinion that this was a unique case of regional achondroplasia of the pelvis and ribs.

Among animals the disease appears both in its complete and its regional form. As an example of the complete form, Irish cattle may be cited, and of the regional form, the bulldog, where the effect appears in the spinal column and the skull (Lane, 1960). Transmission of the disease among some animals such as cattle appears as a dominant trait.

A similar disease has been described in rabbits where transmission is recessive. The homozygote being non-vital dies in utero or shortly after delivery, whereas the heterozygote develops entirely normally (Brown and Pearce, 1945).

In the human, genetic transmission is accepted as being dominant (Mörch, 1940). Evidence of genetic transmission can be ascertained in only $20 \%$ of patients, the remainder being sporadic cases apparently due to spontaneous mutation. The spontaneous mutation rate in this disease has been
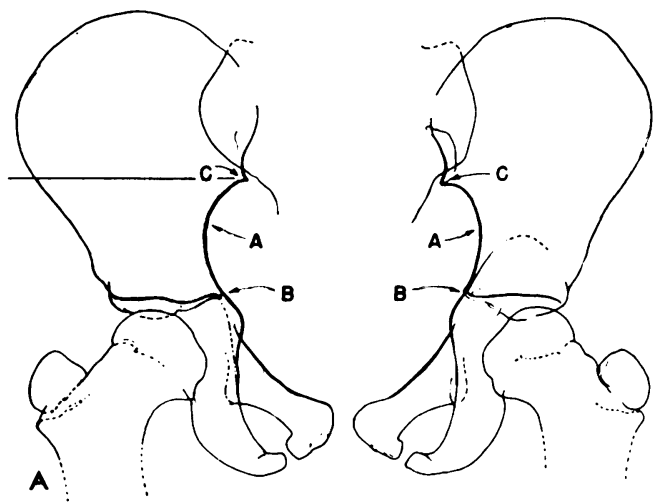

Fig. 5.-Comparison of normal (A) and achondroplastic pelves (B) at 5 years of age. Letters A mark the greater sciatic notches; B the Y cartilages; and $\mathrm{C}$ the postero-inferior iliac spines. In the achondroplast practically all the ilium below these spines is missing and the greater sciatic notches are reduced to narrow deep clefts. Iliums are square with elongated flat lower edges.

(Reprinted from Caffey, J. (1958). Achondroplasia of pelvis and lumbosacral spine; some roentgenographic features. Amer. J. Roentgenol., 80, 449.) 
estimated to be $4 \times 10^{-5}$ or one mutant birth in 12,000 (Mörch, 1941). Catherine de Medici is known to have made unsuccessful attempts to breed achondroplastic dwarfs by intermarriage (Rischbieth, 1912).

In both the complete and the regional forms of achondroplasia, the typical pot-belly is explained by the effect on posture of the changes in the vertebrae and pelvis. A normal development of skeletal muscles is usually emphasized in achondroplastic dwarfs. In our case, the hypoplastic abdominal musculature was the cause of the protuberant abdomen, a feature not previously described.

Another striking feature in this family was the high incidence of herniae. It seems possible, therefore, that the muscular and skeletal defects are related. According to the data available from this pedigree no conclusion about linkage can be drawn, though the mode of inheritance, both of the hernia and the chest deformity, seems compatible with autosomal dominant transmission with incomplete penetrance.

Since chondrodystrophy occurs in phenotypically different forms, different genes may be involved and recessive inheritance could occasionally occur. This could be the case in this family, where consanguinity may have been a factor in producing affected homozygotes in generation IV. The high mortality rate seen in the generation of the patient's father, may have been due to severely affected patients, though the combination of chest deformity with a tendency to recurrent pulmonary infections, which were successfully treated in our patient, may well have been the cause of death in others. However, the high mortality rate could have been the result of poor health conditions in their homeland, the family having migrated later to Israel from a middle-eastern country with a high infant mortality rate.

The gradual improvement over the years in the radiological findings and of the abdominal muscula- ture in our case provides us with a possible explanation for the lack of abnormal radiological findings in both the older sister and the aunt of the patient, in spite of the marked resemblance from the clinical point of view.

\section{Summary}

An infant with extreme hypoplasia of the abdominal muscles and skeletal deformities was found to have achondroplasia of the ribs and ilium. The rest of the skeleton was normal. Follow-up examination at the age of 4 years showed fair development of the abdominal muscles and good growth of the affected bones. Bilateral direct inguinal hernias were repaired at this time.

A study of the family indicated several persons with chest deformity, and $50 \%$ of grown-up survivors with herniae.

Regional achondroplasia of the ribs and ilium is rare, and its occurrence with abdominal muscle weakness as a specific syndrome is reported here for the first time. The pedigree is compatible with autosomal dominant transmission with incomplete penetrance.

We should like to thank Professor John Caffey for reviewing the radiographs.

\section{REFERENCES}

Brown, W. H., and Pearce, L. (1945). Hereditary achondroplasia in the rabbit. J. exp. Med., 82, 241.

Caffey, J. (1948). Achondroplasia. In Practice of Pediatrics, Vol. IV, chap. 28, ed. J. Brennemann. W. F. Prior Co., Hagerstown, Maryland.

(1958). Achondroplasia of pelvis and lumbo-sacral spine. Amer. J. Roentgenol., 80, 449.

Lane, J. W. (1960). Roentgenographic manifestations of the cartilaginous dysplasias. Amer. J. med. Sci., 240, 636.

Mörch, E. T. (1940). Achondroplasia is always hereditary and is inherited dominantly. J. Hered., 31, 439.

(1941). Chondrodystrophic dwarfs in Denmark. Op. Domo Biol. hered. hum., Kbh., 3.

Parrot, M. J. (1878). Sur la malformation achondroplasique et le dieu Ptah. Bull. Soc. Anthrop. Paris, 1 (3rd series), 296.

Rischbieth, H. (1912). Dwarfism. Treas. hum. Inher., 1, 355. 